\title{
Beta-strength and anti-neutrino spectra from total absorption spectroscopy of a decay chain ${ }^{142} \mathrm{Cs} \rightarrow{ }^{142} \mathrm{Ba} \rightarrow{ }^{142} \mathrm{La}$
}

\author{
Marzena Wolińska-Cichocka ${ }^{1,2,3, a}$, B. Charles Rasco ${ }^{2,3,4}$, Krzysztof P. Rykaczewski ${ }^{2}$, Nathan T. Brewer ${ }^{2,3,4}$, \\ Daniel Stracener ${ }^{2}$, Robert Grzywacz ${ }^{2,3,4}$, Carl J. Gross ${ }^{2}$, Aleksandra Fijałkowska ${ }^{5}$, K. Callie Goetz ${ }^{4}$, Marek Karny ${ }^{5}$, \\ Toby King ${ }^{4}$, Shintaro Go ${ }^{4}$, Elizabeth A. McCutchan ${ }^{6}$, Caroline Nesaraja ${ }^{2}$, Alejandro A. Sonzogni ${ }^{6}$, Enhong Wang ${ }^{7}$, \\ Jeff A. Winger ${ }^{8}$, Yongchi Xiao ${ }^{4}$, Christopher J. Zachary ${ }^{7}$, and Edward F. Zganjar ${ }^{9}$ \\ 1 Heavy Ion Laboratory, University of Warsaw, 02-093 Warsaw, Poland \\ 2 Physics Division, Oak Ridge National Laboratory, Oak Ridge, TN 37831, USA \\ 3 JINPA, Oak Ridge National Laboratory, Oak Ridge, TN 37831, USA \\ ${ }^{4}$ Department of Physics and Astronomy, University of Tennessee, Knoxville, TN 37996, USA \\ 5 Faculty of Physics, University of Warsaw, 02-093 Warsaw, Poland \\ ${ }^{6}$ National Nuclear Data Center, Brookhaven National Laboratory, Upton, NY 11973, USA \\ 7 Department of Physics and Astronomy, Vanderbilt University, Nashville, TN 37235, USA \\ 8 Department of Physics and Astronomy, Mississippi State University, Mississippi State, MS 39762, USA \\ 9 Department of Physics and Astronomy, Louisiana State University, Baton Rouge, LA 70803, USA
}

\begin{abstract}
Beta decays of mass $\mathrm{A}=142$ isobaric chain starting from ${ }^{142} \mathrm{Cs}$ have been investigated by means of Modular Total Absorption Spectrometer (MTAS) and on-line mass separation at Oak Ridge National Laboratory. The beta strength distribution derived for ${ }^{142} \mathrm{Cs}$ decay from MTAS spectra is showing significant differences in $\beta$-feeding pattern when compared to the values listed at nuclear databases. MTAS results are shifting the associated anti-neutrino energy spectrum towards lower energies. A decay pattern deduced for ${ }^{142} \mathrm{Ba}$ is similar to earlier reported results.
\end{abstract}

\section{Introduction}

The Modular Total Absorption Spectrometer (MTAS) array has been designed, constructed, characterized and applied to the decay studies of ${ }^{238} \mathrm{U}$ fission products at the Holifield Radioactive Ion Beam Facility (HRIBF) at Oak Ridge National Laboratory [1-3]. MTAS measurements yield true $\beta$-strength distribution verifying and helping to develop the microscopic description of $\beta$-decay. In particular, the decay heat release in fission products during a nuclear fuel cycle as well as respective anti-neutrino energy spectra can be determined from the complete $\beta$-decay scheme. Over seventy decay products of ${ }^{238} \mathrm{U}$ fission products measured at ORNL include 22 decays of high priority for decay heat analysis [4]. These measurements include the ${ }^{142} \mathrm{Cs}$ activity assessed recently as the third most important contributor to the high energy $\bar{v}$ spectra in nuclear reactors [5-7]. The significant differences between $\beta$-feeding values derived from MTAS measurement and Evaluated Nuclear Structure Data Files (ENSDF) database are substantially impacting the reference reactor antineutrino flux and its spectral properties, see, e.g., [8].

\section{The modular total absorption spectrometer}

MTAS measurement campaigns were performed in January 2012, March 2015, October-December 2015 and

a e-mail: mala@slcj.uw.edu.pl
January 2016 at the On-Line Test Facility, namely the mass separator on-line to the ORNL Tandem accelerator. MTAS detector array consists of $19 \mathrm{NaI}(\mathrm{Tl})$ hexagonal shape detectors. Each crystal is $\sim 53 \mathrm{~cm}$ long and about $20 \mathrm{~cm}$ maximum diameter. The weight of $\mathrm{NaI}(\mathrm{Tl})$ detector material in MTAS array is $\sim 1000 \mathrm{~kg}$, covering over 99\% of the solid angle around the measured activities. The entire array is surrounded by over $5000 \mathrm{~kg}$ of lead and SWX-227A neutron shielding foam. This shielding reduces the laboratory gamma background by a factor of 1000. MTAS auxiliary detectors include two 1 -mm-thick $\mathrm{Si} \beta$-trigger detectors (each divided into 7 strips, each $\sim 8.5 \mathrm{~mm}$ wide), placed at the center of the $\mathrm{NaI}(\mathrm{Tl})$ array around the tape transporting the activities [1]. The signals detected in these silicon counters are used as $\beta$-triggers. Applying coincidence condition between silicon detectors and MTAS suppresses typical laboratory background by at least three orders of magnitude. The $\gamma$-ray efficiency for full-energy deposition of a single $\gamma$-ray in on-line conditions is about $81 \%$ at $300 \mathrm{keV}$ and about $71 \%$ at $6 \mathrm{MeV}$ [3]. The MTAS thresholds for individual detector, each from 18 regular modules, is about $25 \mathrm{keV}$. For the central module it is around $30 \mathrm{keV}$.

\section{MTAS measurement and ENSDF database comparison}

We evaluate the energy spectra measured with MTAS to determine $\beta$-feeding intensities to known and unknown

(C) The Authors, published by EDP Sciences. This is an Open Access article distributed under the terms of the Creative Commons Attribution License 4.0 (http://creativecommons.org/licenses/by/4.0/). 
Table 1. The intensities of $\beta$-transitions in ${ }^{142} \mathrm{Ba}$ decay determined from MTAS measurement in comparison to the value from ENSDF and TAGS results of Ref. [14].

\begin{tabular}{|c|c|c|c|}
\hline level energy [keV] & $\mathbf{I}_{\beta}$ ENSDF & $\mathbf{I}_{\beta}$ TAGS [11] & $\mathbf{I}_{\beta}$ MTAS \\
\hline 225.3 & $3.5(7)$ & 4.6 & 3.8 \\
\hline 425.0 & $5.4(2)$ & 6.0 & 5.8 \\
\hline 1204.3 & $46(2)$ & 46 & 45.8 \\
\hline
\end{tabular}

energy levels in the daughter nuclei, including the ground state. The evaluation method is based primarily on the techniques applied in the analysis of other total absorption spectrometers spectra, see e.g., [9-11]. However, our analysis is profiting from the increased efficiency and high segmentation of MTAS [2,3,8]. The MTAS detector response function was simulated with the GEANT4 toolkit. This generated MTAS response function is verified with through measurements of single $\gamma$-ray and $\gamma$-cascades emitted in the decay of calibration sources like ${ }^{137} \mathrm{Cs},{ }^{54} \mathrm{Mn},{ }^{65} \mathrm{Zn},{ }^{60} \mathrm{Co}$ and ${ }^{24} \mathrm{Na}$. MTAS energy spectra are divided into two response regimes. These correspond to energy levels below threshold energy with de-excitation $\gamma$-paths known from high energy resolution gamma spectroscopy data and to unknown $\beta$-fed levels above the threshold energy grouped in $25 \mathrm{keV}$ wide bins. More details of MTAS spectra analysis method are given in $[1-3,8]$. In this paper we present MTAS results on measured ${ }^{142} \mathrm{Cs}$ and ${ }^{142} \mathrm{Ba}$ activities compared to GEANT4 simulations using currently available ENSDF entries [12]. Beta-gated MTAS spectra are analyzed to reduce background. Also the pile-up analysis is included into our evaluation procedure [2]. The decay chain of the $\mathrm{A}=142$ includes the very short-lived ${ }^{142} \mathrm{Cs}(1.7 \mathrm{~s})$ isotope which decays to ${ }^{142} \mathrm{Ba}$ activity having a half-life of $10.6 \mathrm{~min}$, followed by the decay of ${ }^{142} \mathrm{La}(91.1 \mathrm{~min})$ to stable ${ }^{142} \mathrm{Ce}$.

\subsection{Decay of ${ }^{142} \mathrm{Ba}$}

There were several measurements of ${ }^{142} \mathrm{Ba}$ decay, see, e.g., [13], and summarized recently in Nuclear Data Sheets [12]. These measurements were done using highresolution Ge detectors as well as total absorption gamma spectroscopy (TAGS) [14]. Interestingly, the ${ }^{142} \mathrm{Ba}$ decay is a rare case where the high-resolution measurements point to practically the same $\beta$-decay pattern as deduced from the TAGS measurement. It could be explained by a relatively low beta decay energy value of $\mathrm{Q}_{\beta}=$ $2212(5) \mathrm{keV}$. Indeed, ${ }^{142} \mathrm{Ba}$ MTAS spectrum is in general agreement with earlier data (Fig. 1). Figure 2 presents the comparison of an overall fit of the simulated decays following the ENSDF entry for the ${ }^{142}$ La level scheme populated in ${ }^{142} \mathrm{Ba}$ decay (colored components) and their sum (orange), to the measured MTAS spectrum (black). The sum of the individual decay paths follows well MTAS measured data. The $\gamma$-rays with intensity much smaller then $10 \%$ of the strongest $\gamma$-ray de-exciting each level were not taken into account in this particular simulation.

The intensities of $\beta$-feeding determined from MTAS measurement were compared to the values from ENSDF entries and Greenwood's measurements [14]. Examples of the results for main levels are listed in Table 1.

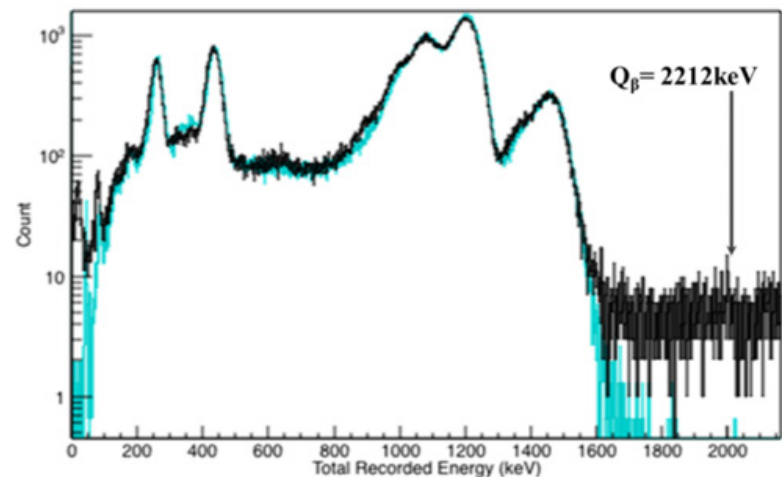

Figure 1. Background subtracted ${ }^{142} \mathrm{Ba}$ MTAS data (black) compared to the simulated MTAS response for ${ }^{142} \mathrm{Ba}$ decay events based on the ENSDF entry (cyan).

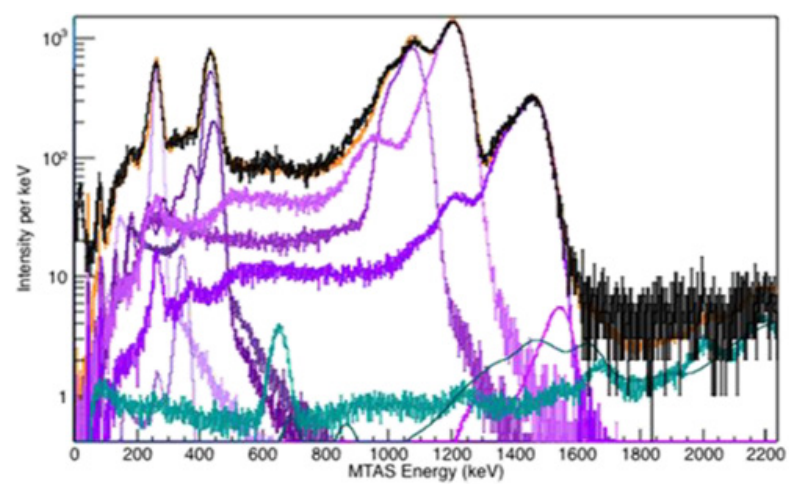

Figure 2. Fit of simulated $\gamma$-ray response functions (colored) for complex decays of individual levels to the total ${ }^{142} \mathrm{Ba}$ $>142$ La MTAS decay data (black). The sum of all the simulated components (orange) is in good agreement with an experimental spectrum.

\subsection{Decay of ${ }^{142} \mathrm{Cs}$}

The MTAS-measured energy spectrum for ${ }^{142} \mathrm{Cs}$ decay is compared to the simulated MTAS response using decay data listed in the current ENSDF entry in Fig. 3. The reduction of the ground-state $\beta$-feeding and the $\beta$-feeding to the first-excited $2+$ state at $360 \mathrm{keV}$ is clearly visible, as well as the presence of new $\beta$-fed levels at higher excitation energies. The derived $\beta$-feeding intensities for ${ }^{142} \mathrm{Cs}$ decay with $\mathrm{Q}_{\beta}=7325(9) \mathrm{keV}$ are presented in Fig. 4. The MTAS-determined ${ }^{142} \mathrm{Cs} \beta$-feeding to the ${ }^{142} \mathrm{Ba}$ ground-state is $44(2) \%$, lower than $56 \%$ listed in ENSDF. The upper limit for beta-feeding to the first-excited $2+$ state in ${ }^{142} \mathrm{Ba}$ is $0.5 \%$ [8], at least an order of magnitude lower than reported earlier $7 \%$ [12].

\section{High energy part of reactor anti-neutrino spectrum for ${ }^{142} \mathrm{Cs}$}

The ${ }^{142} \mathrm{Cs}$ beta decay was found to be among top 3 contributors to the high energy reactor $\bar{v}$ spectrum in recent evaluations [5-7]. We have re-evaluated the spectrum of anti-neutrinos emitted in this decay basing on new MTAS data. The change in the $\bar{v}$ energy spectrum of ${ }^{142} \mathrm{Cs}$ is shown in Fig. 5. The fraction of $\bar{v}$ with energy above $5 \mathrm{MeV}$ changes from $20 \%$ to $14(1) \%$ the total $\bar{v}$ flux from ${ }^{142} \mathrm{Cs}$ decay, while the fraction of ${ }^{142} \mathrm{Cs} \bar{v}$ with energy below $1.8 \mathrm{MeV}$ increases from $11 \%$ to $23(3) \%$ 




Figure 3. Background subtracted ${ }^{142} \mathrm{Cs}$ MTAS data (black) compared to the simulated MTAS response to ${ }^{142} \mathrm{Cs}$ decay events based on the ENSDF data (cyan), see [8].

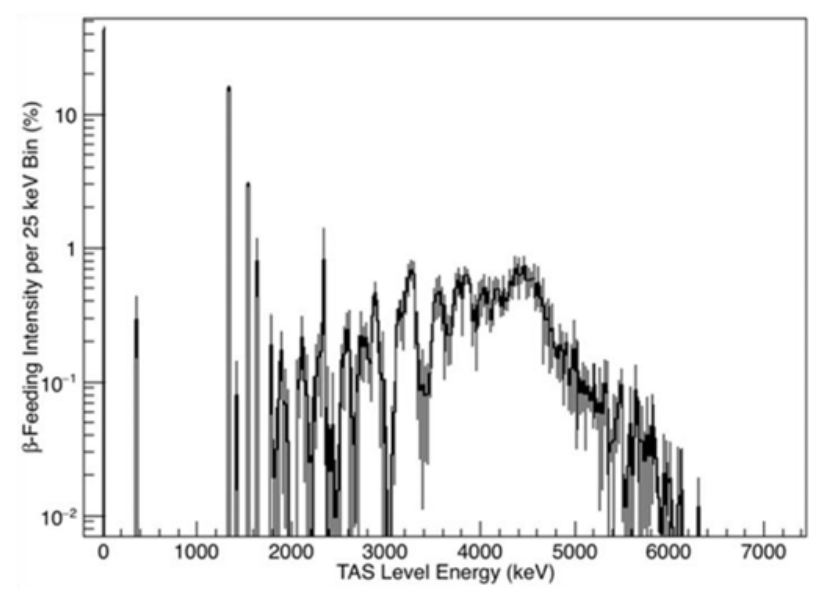

Figure 4. Average ${ }^{142} \mathrm{Cs} \beta$-feeding intensity with the uncertainty based on the number of $\gamma$-rays in the de-excitation cascade from each level. The fit for the ground state $\beta$-feeding is at $44(2) \%$.

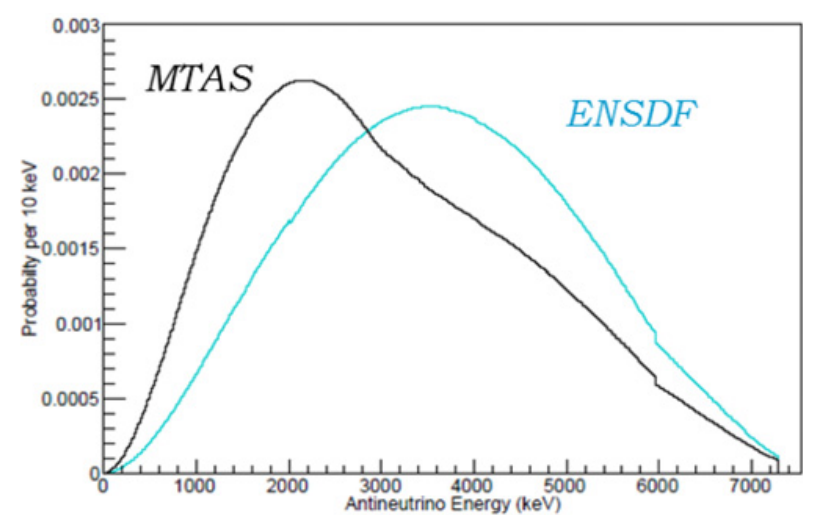

Figure 5. ${ }^{142} \mathrm{Cs} \bar{v}$ energy spectrum derived from MTAS data (black) compared to the $\bar{v}$ energies obtained from the latest ENSDF entry (cyan).

[8]. Such change can be expected for complex decays of fission products, with high Q-value and influenced by the Pandemonium Effect [15] after the measurement using the total absorption technique. It shows the reduction of previously reported feeding to low-lying states and a corresponding increase of $\beta$-strength at higher excitations in the daughter nucleus. It shifts the corresponding $\bar{v}$ spectrum to lower energies.
These changes reduce the number of anti-neutrino interactions with matter. The modification of ${ }^{142} \mathrm{Cs}$ decay scheme alone reduced the reported reactor $\bar{v}$ anomaly of 94.6(22)\% [16] to 95.7(22)\%. Simultaneously, it increased the high-energy "shoulder" from $\sim 10 \%$ to $\sim 12 \%$ excess, compare $[5-8,16]$.

\section{Summary}

The Modular Total Absorption Spectrometer (MTAS) was used to measure the beta-gamma activities in the mass $A=142$ decay chain. Two isobars from this chain, ${ }^{142} \mathrm{Ba}$ and ${ }^{142} \mathrm{Cs}$, are presented here. For both of them, the new $\beta$-feeding pattern was obtained. For ${ }^{142} \mathrm{Ba}$ decay, with low $\mathrm{Q}_{\beta}$ of $2.2 \mathrm{MeV}$, MTAS spectra analysis reproduce known decay pattern obtained previously using high resolution gamma spectroscopy as well as total absorption measurements [14]. MTAS measurements of ${ }^{142} \mathrm{Cs}$, with much higher $\mathrm{Q}_{\beta}$ of $7.3 \mathrm{MeV}$, resulted in a major modification of the decay scheme. The groundstate $\beta$-feeding is reduced from $56 \%$ (ENSDF) to $44(2) \%$ (MTAS). For the $\beta$-feeding to the first-excited $2^{+}$state at $360 \mathrm{keV}$ only a limit of $\beta$-feeding $\sim 0.5 \%$ can be given, while the ENSDF value is listed as $7 \%$. Our measurements are showing also the presence of new $\beta$-fed levels at higher excitation energies in ${ }^{142} \mathrm{Ba}$. MTAS measurements confirmed the importance of ${ }^{142} \mathrm{Cs}$ decay as one of the top three contributors to the high energy $\bar{v}$ spectral shape in nuclear reactors, and as so far the most important activity changing the expected high-energy anti-neutrino flux. MTAS results for ${ }^{142} \mathrm{Cs}$ shift the total $\bar{v}$-spectrum toward lower energies. It results in a reduction of reactor $\bar{v}$ anomaly, roughly from $94.6(22) \%$ to $95.7(22) \%$ and is enhancing high-energy "bump" from $\sim 10 \%$ to $\sim 12 \%$ excess, for the reference anti-neutrino flux [8] calculated using a summation method.

We would like to thank the ORNL Tandem operations staff for providing the excellent quality proton beams necessary for this work. This research was also sponsored by the Office of Nuclear Physics, U. S. Department of Energy under contracts DE-AC05-00OR22725 (ORNL), DE-FG02-96ER40983 (UTK), DE-FG02-96ER40978 (LSU), DE-FG02-96ER41006 (MSU), DE-FG-05-88ER40407 (VU), and by the Polish National Science Center under contracts UMO-2015/18/E/ST2/00217 and UMO2013/08/T/ST2/00624.

\section{References}

[1] M. Wolińska-Cichocka, K.P. Rykaczewski, A. Fijałkowska, M. Karny, R.K. Grzywacz, C.J. Gross, J.W. Johnson, B.C. Rasco, E.F. Zganjar, NDS 120, 22 (2014)

[2] B.C. Rasco, A. Fijałkowska, M. Karny, K.P. Rykaczewski, M. Wolińska-Cichocka, R. Grzywacz, K.C. Goetz, NIM A 788, 137 (2015)

[3] M. Karny, K.P. Rykaczewski, A. Fijałkowska, B.C. Rasco, M. Wolińska-Cichocka, R.K. Grzywacz, K.C. Goetz, D. Miller, E.F. Zganjar, NIM A 836, 83 (2016)

[4] "Assessment of fission product decay data for decay heat calculations" OECD 2007, NEA No. 6284, 25, ISBN 978-92-64-99034-0

[5] A.A. Sonzogni, T.D. Johnson, E.A. McCutchan, PR C 91, 011301 (2015) 
[6] D.A. Dwyer, T.J. Langford, PRL 114, 012502 (2015)

[7] A.-A. Zakari-Issoufou et al., PRL 115, 102503 (2015)

[8] B.C. Rasco, M. Wolińska-Cichocka, A. Fijałkowska, K.P. Rykaczewski, M. Karny, R.K. Grzywacz, K.C. Goetz, C.J. Gross, D.W. Stracener, E.F. Zganjar, J.C. Batchelder, J.C. Blackmon, N.T. Brewer, S. Go, B. Heffron, T. King, J.T. Matta, K. Miernik, C.D. Nesaraja, S.V. Paulauskas, M.M. Rajabali, E.H. Wang, J.A. Winger, Y. Xiao, C.J. Zachary, PRL 117, 092501 (2016)

[9] D. Cano-Ott, J.L. Taín, A. Gadea, B. Rubio, L. Batist, M. Karny, and E. Roeckl, NIM A 430, 333 (1999)
[10] J.L. Taín and D. Cano-Ott, NIM A 571, 728 (2007)

[11] A. Simon, S. Quinn, A. Spyrou, A. Battaglia, I. Beskin, A. Best, B. Bucher, M. Couder, P. DeYoung, X. Fang et al., NIM A 703, 16 (2013)

[12] T.D. Johnson et al., NDS 112, 8 (2011)

[13] M. Wang et al., Chin. Phys. C 36(12), 1603 (2012)

[14] R.C. Greenwood, R.G. Helmer, M.H. Putnam, K.D. Watts, NIM A 390, 95 (1997)

[15] J.C. Hardy, L.C. Carraz, B. Jonson, P.G. Hansen, Phys. Lett. B 71, 307 (1977)

[16] F.P. An et al. (Daya Bay Collaboration), PRL 116, 061801 (2016) 